the university library possesses a portrait of him, which is attributed to Rubens. His most famous secret remedy got after his death into the possession of the Geneva physician Colladon. Detailed biographical notes of this interesting man who published the Theatrum Insectorum in London in 1634 can be found in the "Biographie générale», Paris (1860), vol. 33 , p. $544-546$.

\title{
Die Augsburger erneuerte Hebammenordnung von 1750
}

Alte Hebammenordnungen und «Hebammenschulen» sind in größerer Zahl erhalten geblieben, ich erinnere an diejenige des Eucharius Roeßlin (1513), des Ortollfus (1500); etwas später erschien der «Rosengarten» des Straßburger «Chirurgen» Walter Reiff, (Rivius) von 1545, dann jener von Steinschneider (1554). Dann kam das bedeutend wertvollere «Trostbüchli» des Zürchers Jakob Rueff (1554). Im 17. Jahrhundert folgte die «Hebammenschul» des Christoph Völtern in Württemberg (1627), die «Chur-Brandenburgische Hof-Wehe-Mutter» der Justine Siegemundin (1690), die "Morgenröte der Hebammen» (1696) Hendrik van Deventers (1651-1724); im 18. Jahrhundert endlich neben vielen andern die «Helvetisch-Vernünftige Wehemutter» des Johann Fatio (1752). Sie alle teilen vielfach das Schicksal ihrer nachgeborenen Schwestern bis auf unsere Tage, insofern sie nämlich mehr oder minder gleich lauten und in irgendeiner Form schon Gesagtes wiederbringen. Die Augsburgerordnung vom Jahre 1750 macht insofern eine Ausnahme, als sie durch Eigenart und Umfang besonders medizin-historisches Interesse beanspruchen darf. Es ist tatsächlich ein kleines Buch von nicht weniger als 95 Seiten, um das es sich hier handelt, das auf alle Details und kasuistischen Möglichkeiten weitgehend Rücksicht nimmt und uns damit einen wertvollen Einblick vermittelt in die hohe Bedeutung, die schon vor $200 \mathrm{Jahren}$ einsichtige Behörden einer zweckmäßigen Geburtshilfe beimaßen. Gedruckt ist der Erlaß beim Augsburger Stadtbuchdrucker Andreas Bringhausser. Die amtliche Gutheißung ist datiert vom 1. Juli 1749. Sie nimmt ausdrücklich Bezug auf eine «bisherige der Verbesserung wohl nötige Verordnung» und richtet sich sowohl an die «liebe Burgerschaft» als an «die obrig- 
keitlich verordnete jederweilige Ob-Frauen, Herren Doktores, die angenommene und bestellte Hebammen, Accoucheurs und wer in Geburten beisteht».

Die Institution der «Ob-Frauen» erscheint hier als neu. Was hat man darunter zu verstehen? Nach Grimm sind sie bestimmt «zur Obsicht und Direktion über die Hebammenverordnung». Er beruft sich bei dieser Angabe ausschließlich auf die in Rede stehende Augsburger Hebammenordnung vom Jahre 1750. Es mag interessieren, daß sich in gewissem Sinne übrigens eine ähnliche Institution bis auf unsere Tage erhalten hat, wenn auch vielleicht nicht genau mit derselben Mission, aber doch an diese anklingend. Aus meiner eigenen Tätigkeit als früherer, langjähriger Hebammenlehrer, dem auch die Ausbildung der thurgauischen Hebammen anvertraut war, ist mir bekannt gewesen, daß dort die Hebamme, nicht etwa wie anderswo, entweder frei sich niederlassen konnte oder von der Behörde bestimmt wurde, sondern die Kandidatinnen mußten jeweils zu Handen der in den betreffenden Gemeinden oder Distrikten amtenden Frauengemeinde begutachtet werden.

Ich habe mich deshalb an das thurgauische Sanitätsdepartement mit der Frage gewandt, ob dieser Wahlmodus auch heute noch seine Gültigkeit habe und erhielt darauf in verdankenswerter Weise die Antwort, daß im Kanton Thurgau die Wahl der Hebammen durch die Frauen bis zum Erlaß der neuen Hebammenverordnung vom 20. Juli 1943 durch die sogenannte Frauengemeinde erfolgte. Stimmberechtigt waren die Frauen der Munizipalgemeinden und zwar die Verheirateten und die Verwitweten. Sie wurden zur Hebammenwahl einberufen vom Gemeindeammann, Gemeindepräsident, der auch die Verhandlungen leitete. Eine Hebamme wurde nur einmal gewählt. Es gab keine periodische Wiederwahl. Eine von der Frauengemeinde gewählte Hebamme blieb in ihrem Amt, solange sie nicht demissionierte oder von der Gesundheitskommission bzw. den Sanitätsbehörden abberufen wurde. Die neue thurgauische Verordnung bestimmt nunmehr die Wahl der Hebammen durch die Gesundheitskom. mission der Munizipalgemeinden. Wir sehen also, daß sich ein ähnliches Institut, wie das der Ob-Frauen, in gewissem Sinne bis auf unsere Tage erhalten hat.

Auffällig und einzig in ihrer Art an der A. O. (Augsburger Ordnung) ist, daß sie sich ausdrücklich an die «Hebammen allhier beider Religionen» richtet. Diese strenge Trennung finden wir später bis in die kleinste Anordnung hinein durchgeführt, worüber noch zu sprechen sein wird. 
In dem einleitenden Artikel über die Bedeutung ihrer Aufgabe wird die Hebamme ausdrücklich auch als Rechtshelferin bezeichnet, indem sie zu Handen der Behörde sich aussprechen muß über die Tüchtigkeit oder Untüchtigkeit zur Ehe, uneheliche Schwangerschaft, heimliches Gebären, Abtreiben der Leibesfrucht, unzeitige oder frühzeitige Geburt, Kindsmord und andere «der Justiz unterworfene Begebenheiten». Ein außerdem in solchen Fällen einzuholendes ärztliches Gutachten wird ausdrücklich erwähnt. Überhaupt wird der Hebammenberuf als ein besonders hoher und segensreicher dargestellt und der gewissenhaften Hebamme auch «Gottes Beistand und Segen nach dem schönen Exempel der gottesfürchtigen und von Gott gesegneten israelitischen Hebammen Siphra und Pua im I. Kap. des II. Buch Moses» in Aussicht gestellt.

Einzigartig ist die Einteilung der Hebammen nach folgenden Kategorien: Vier lernende, neun besoldete geschworene Hebammen; auch eine ins Brechhaus oder Lazarett und eine andere "vors Tor für die Leute so draußen wohnen und vier Führerinnen». Auch bei den Schülerinnen begegnet uns die strenge konfessionelle Trennung: Zwei sollen «der katholischen Religion und zwei der Augsburgischen Konfession» zugetan sein. Die Ausbildungszeit beträgt drei Jahre bei einer ältern Hebamme, die sich als besonders geschickt erwiesen oder aber einer besonders bestellten, sogenannten «Führerin». Darauf folgt die Prüfung und Vereidigung. Aber nur bei Ableben einer besoldeten Hebamme wird sie nach und nach in die Besoldung aufgenommen. Denn erst die «examinierten und geschworenen» Hebammen der Stadt sind berechtigt, ihren erlernten Dienst auszuüben. Es gab, wie bereits erwähnt, deren neun und zwar wiederum in strenger Trennung, fünf der Augsburger Konfession und vier der katholischen Religion. Es kann in diese Klasse der geschworenen Stadthebammen auch eine ausgelernte und im Examen gut befundene Schülerin zwar aufgenommen, aber nicht sofort besoldet werden. Neben diesen neun besoldeten Hebammen werden zwei ebenfalls Examinierte mit besonderer Aufgabe betreut, die eine hat hauptsächlich das Blatternhaus zu besorgen, die andere das Nothaus vor dem Tor, wo sich die Nichteingebürgerten, fahrendes Volk usw. aufzuhalten hatten. Auch hier ist die konfessionelle Trennung streng durchgeführt, indem jede dieser beiden Hebammen immer eine Schülerin der andern Konfession bei Geburten mitzunehmen hat, damit auf jeden Fall die konfessionelle Betreuung gewährleistet bleibt.

Die spezielle Pflichtordnung unterscheidet in erster Linie die Pflichten 
gegen Gott, die Anrufung seines Beistandes, die Vermeidung allen Aberglaubens, die Bekämpfung unnützer Gewohnheiten und Sprüchlein und sündiger Gebräuche.

Darauf erst folgt die Aufzählung der Pflichten gegenüber der Obrigkeit. An deren Spitze steht das Bauamt, dem die Hebamme «allzeit untertänigst und gehorsam sein muß. Ohne Erlaubnis darf sie sich nicht aus der Stadt entfernen und muß ihr Quatember-Geld am Samstag oder, wenn ein Feiertag einfällt, am Freitag nach dem Quatember nachmittags von den Bauherrn selber holen in ehrbaren Kleidern.» Im Verhinderungsfalle muß sie sich durch eine Mithebamme beim Bauamt entschuldigen. Auch gegenüber den Ob-Frauen ist ihre Pflicht genau geregelt, ebenso wie im Verhältnis zu den «zur Hebammen-Ordnung verordneten Herren Doktores». Daraus erhellt ohne weiteres das Bestehen einer Art ärztlicher Aufsichtskommission über das Hebammenwesen. «Den ObFrauen und Herren Doktores soll sie nicht übel nachreden oder ihrem Urteil und Zurechtweisung sich widersetzen oder ihr gutes, vernünftiges Unterrichten, Bedenken und Rat, als ob sie es besser verstünden, weder unters Angesicht noch hinterrucks bei andern Leuten tadeln.»Dafür hat die Hebamme, "wenn sie ohne Verschulden untereinander oder von andern Personen verklagt oder sonst ihrer Verrichtungen halber angefochten wird, von Seiten der Herren Doktores und Ob-Frauen gewissenhafte Fürsprechung, Verteidigung, guten Rat und Hülfe in alle Wege zu gewarten». Damit ist aber die Reihe der Respektspersonen keineswegs erschöpft, sondern es folgt weiter eine genaue Anweisung über das Verhalten gegenüber dem Herrn «Dekan, Vikar und übrigen Herren Doktores des Collegii Medici», von denen sie aber keinen beim nötigen $\mathrm{Zu}$ zug eines Arztes bevorzugen darf, «sondern den Leuten ihre selbsteigene Wahl und Willkür frei lassen soll». Eine weitere Klasse betrifft die «Accoucheurs oder Geburtshelfer und Operateurs». Hier scheint es sich um eine offenbar niedrigere Klasse von Heilbeflissenen zu handeln, denn sie reagieren unter den Doktores. Wahrscheinlich hat man dabei an Laien zu denken, deren Zuzug die Bevölkerung in erster Linie verlangt, etwa so wie wir das ja in der Geburtshilfe der Haustiere heute noch erleben, wo immer zuerst vor dem Tierarzt eine für ihre geburtshilfliche Kunst besonders angesehene Persönlichkeit aus dem Laienstande bevorzugt wird. Die Richtigkeit dieser Vermutung ergibt sich schon aus dem Umstande, daß auch rein äußerlich in der Titulatur ein deutlicher Unterschied gemacht wird: Zwischen Herrn Doktores und den gewöhnlichen 
Geburtshelfern und Operateurs, die als solche schlechthin bezeichnet werden, ohne Beifügung des Titels «Herrn». Die Funktion dieser Art Geburtshelfer war denn auch ausdrücklich beschränkt. Die Hebamme soll zwar, "wenn ein solcher zu harten Geburten kommt», seiner guten Anweisung folgen und nötigenfalls ihn «selbst die Hand ans Werk legen, jedoch nichts Gefährliches tun lassen».

«Alle Vierteljahre haben sämtliche drei Kategorien von Hebammen an bestimmtem Ort sich einzufinden, um zunächst eine Art Prüfung zu bestehen und zwar soll, eine um die andere von Lernerinnen einen Jahrgang hindurch ansagen'.» Sie haben auch zu referieren über schwere Fälle und eventuelle Klagen gegeneinander vorzubringen. Bei dieser Gelegenheit wird jeweilen, und zwar auch wieder «von jedem Religionsteil besonders, ,eine Geldbüchs' aufgestellt, zur Sammlung von Gaben für dürftige, gebrechliche, erkrankte Hebammen und ihrer Kinder und Waisen.» Ein bemerkenswerter sozialer Anklang an den Plan einer Kranken-, Alters- und Hinterbliebenenversicherung. Der Beitrag beträgt für alle Hebammen-Kategorien einen Groschen. Auch Strafgelder werden dieser Kasse zugeführt. Die älteste Ob-Frau führt Rechnung und die jüngste bewahrt den Schlüssel auf.»

Verboten ist Hebammen und Schülerinnen jegliche andere Berufsbetätigung, wie das Hochzeitladen, das Leichensagen, das Kinderwarten, das Aufwarten in Bädern, die Hilfe bei Wäschen und beim «Häuser-Ausbutzen», zwecks Schonung ihrer Hände. Beim Verlassen des Hauses ist genau anzugeben, wo sie anzutreffen sei und beim Wegbleiben über Nacht soll sie das «den Ob-Frauen ihrer Religion» vorher anzeigen.

Die Aufnahmebedingungen in die Klasse der Lernenden sind mit sehr vielen Klauseln belastet. Die Altersgrenze beträgt 25 bis 45 Jahre. Nur Frauen, die selber «Kinder getragen, geboren, verpfleget und aufgezogen und zum Hebammendienst einen guten Trieb, Lust und innerlichen Beruf haben", werden zugelassen. Daneben soll die Kandidatin gottesfürchtig, ehrbar, unbescholten sein, über guten Verstand und Gedächtnis verfügen und «unverletzte Sinnen an Hören und Sehen aufweisen». Gewissenhaft, arbeitsam, nüchtern, züchtig, bescheiden, verschwiegen, friedsam, sanftmütig und geduldig wird sie gewünscht, «an Gliedern und Armen nicht großbeinig, sonderlich an Händen reinlich, geschmeidig und gelenk und mit keiner unreinen oder schreckhaften Krankheit belastet». Gedrucktes und Geschriebenes muß sie fertig lesen und schreiben können. Über das Bestehen all dieser körperlichen und geistigen Vorzüge wachen 
bei der Aufnahme die Ob-Frauen und «der zur Hebammen-Ordnung verordnete Herr Medicus ihrer Religion", die darüber dem zuständigen Bauamt Bericht erstatten. Endlich hat sie noch von ihrem Herrn Beichtvater ein schriftliches Zeugnis mitzubringen darüber, daß sie die Nottaufe technisch richtig zu applizieren versteht.

Neben der bereits erwähnten praktischen Anlernung durch die Führerin dienen aber auch zwei Doktores als Lehrer und zwar wieder von jeder Konfession einer. Der Unterricht erfolgt monatlich wenigstens ein paarmal. Die Schülerin darf Lehrer und Lehrerin niemals «tadeln oder darwider reden, sondern soll ihren Rat bescheiden annehmen, ins Gedächtnis fassen und sich darüber aufklären lassen».

Die Instruktion geht aber noch weiter. Auffällig ist die Vorschrift, die Schülerin müsse «zur Erkenntnis des Frauenleibes durch Einsicht toter Leiber kommen». Als Demonstrationsmaterial sind Leichen in der Schwangerschaft oder unter der Geburt bzw. im Wochenbett Verstorbener bevorzugt. Es wird aber der Hebamme zur Pflicht gemacht, bei solchen Todesfällen von den Angehörigen die Eröffnung des Leichnams zu erbitten und «zu wegen bringen unter Vorstellung, wie notwendig zur Offenbarung dunkler und zweifelhafter Umstände, wie dienlich zu der Hinterlassenen eigenen Beruhigung und Vergewisserung und wie nützlich auf andere eben dergleichen Begebenheiten es sei». Auch wenn ein anderer Arzt bei der Geburt tätig war, muß der zum Unterricht Delegierte von der Hebamme zur Sektion gebeten werden, um die Gelegenheit zur Ergänzung des Unterrichts zu benützen. Ihre Kolleginnen soll sie dazu aufbieten.

Nie darf die Schülerin allein eine Geburt übernehmen, sondern nur mit der «Lehrfrau». Sie darf zwar ein Hebammen-Schild an ihrer Haustür anmachen, jedoch ohne das Stadtwappen. Ledige und unehlich Schwangere müssen dem geheimen Strafamt angezeigt werden. Große Bedeutung wird der Ausscheidung und Betreuung sog. «unreiner» Schwangern und Gebärenden beigemessen. Es handelt sich dabei hauptsächlich um die Pocken. Darum muß die Hebamme jede Frau, «ehe sie dieselbe mit einem Finger anrührt, zuvor an ihrem Frauenleib besichtigen, ob sie nicht etwa Merkmale der unreinen Krankheit an sich hat». Im Verdachtsfalle hat die Hebamme des Blatternhauses den Fall zu übernehmen.

Kulturhistorisch äußerst interessant ist dann die vorgeschriebene Pflicht bei der außerehelich Gebärenden, «mit Ernst in sie zu setzen und zu fragen, wer der Kindsvater sei mit Andeuten, daß sie, bevor sie dieses 
wisse, gar nicht Hand anlegen dürfe». Im Falle eines negativen Ergebnisses dieser Inquisition darf sie zwar die Geburt dennoch leiten, aber unter Anzeige an das geheime Strafamt. Wir haben es hier mit dem damals rechtlich sehr wohl umschriebenen und in der Rechtsgeschichte bekannten sogenannten «Niesverhör» zu tun («niesen»= ausnützen). Heute mag uns diese Einrichtung als mit unserem Denken und Fühlen unvereinbar, ja ihm direkt zuwiderlaufend und zu Protest veranlassend dünken. Aber es sind noch ganz wenige Jahre her, erhielt ich von einer Landesregierung eines unserer Kantone ziemlich wörtlich eine gleichlautende Aufforderung. $\mathrm{Da} ß$ ich sie kategorisch ablehnte, ist wohl überflüssig zu sagen, aber Tatsache bleibt, daß sich solche Gedankengänge bis heute offenbar aus naheliegenden, fiskalischen Gründen erhalten haben.

Nach der dreijährigen Ausbildungszeit erfolgt die Prüfung vor zwei verordneten Doktores und den beiden Ob-Frauen. Wiederum ist auch hier die Trennung streng konfessionell, von beiden Seiten je eine Vertretung. Das Examen soll "scharf sein und über die schwersten Geburtsfälle und was die Hebamme sonst recht zu verstehen und zu praktizieren wissen solle», sich erstrecken. Alle im Amt sich befindenden Hebammen müssen der Prüfung beiwohnen, dürfen aber weder heimlich noch laut der Kandidatin einflüstern. Bei mangelhaft bestandener Prüfung kann das Bauamt die Kandidatin entweder ganz abweisen oder «sie zu ihrer Besserung und andern zur Warnung wieder in die Lehre auf eine gewisse Zeit zurückversetzen». Die Diplomierung, welche die Erlaubnis zur Führung eines Hebammen-Schildes mit dem Stadtwappen mit sich bringt, versetzt die bisherige Schülerin nach Ablegung des Amtseides in die Kategorie der «geschworenen Hebamme». Die Vereidigung hat direkt sakralen Charakter. Nochmals folgt eine wörtliche Aufzählung all der genannten Tugenden, auf welche die Verpflichtung lautet, wobei auch das kollegiale Verhalten besonders angedungen wird, denn «es soll immer eine der andern treulich beistehen und beiraten und nichts aus Neid oder Abgunst verschweigen». Auch der Fall ist genau geregelt, daß die für eine Geburt bestellte Hebamme momentan bei einer andern engagiert wäre und eine Stellvertreterin beigezogen werden muß. Es wird den beiden, falls sie schließlich zusammentreffen sollten, zur strengen Pflicht gemacht, jegliche abfällige Bemerkung zu unterlassen. Die Stellvertreterin hat sich bei Ankunft der ursprünglich bestellten entweder zurückzuziehen oder mit der andern in friedlichem Einvernehmen gemeinsam die 
Geburt zu leiten. Wer von den beiden das Kind dann zur Taufe tragen darf, darüber entscheiden die Gebärende und ihre Angehörigen. Auch der Kundenfang ist streng verpönt, und die Pflichten gegen Schwangere, Gebärende und Wöchnerinnen sind nochmals bis in die kleinste Einzelheit ausführlich aufgezählt. Wird eine Hebamme auf die gleiche Zeit zu zwei Geburten bestellt, dann gebührt die Priorität der Schwangern, die sich zuerst meldete, ohne Unterschied von Name, Rang und Stand, während die später sich meldende einer Kollegin abzutreten ist. Im Ablehnungsfalle, d.h. wenn die betreffende Schwangere unter allen Umständen den größten Wert darauf legt, gerade von ihrer Hebamme betreut zu werden, so mag diese zunächst einmal auf Zusehen hin sich bereit erklären, aber unter der ausdrücklichen Bedingung, bei gleichzeitigem Geburtseintritt sie einer Kollegin abtreten zu müssen. Streng verboten ist es, eine Gebärende zu verlassen, um einer zweiten beizustehen. Wir sehen hier eine Fülle von Bestimmungen, wie sie in dieser oder jener Form zum Teil fast wörtlich in die heutigen Pflichtordnungen übergegangen sind.

Die Nachgeburtsbehandlung erfolgt durchwegs nach den Auffassungen der damaligen Zeit: "wo sie nicht bald von selber folget, soll sie die Plazenta mit der Hand abledigen und hinwegnehmen», also in weitgehendem Maße manuelle Plazentarlösung.

Genau geregelt wird das Verhalten der Hebamme bei allen «schweren Geburten» unter Aufzählung aller Regelwidrigkeiten: Wehenschwäche, vorzeitiger Blasensprung, Plazenta praevia, Mißverhältnis zwischen Kopf und Becken, Vorfall kleiner Teile und der Nabelschnur, regelwidrige Lagen und Stellungen, Zwillingsgeburten. Die prophylaktische innere Wendung mit angeschlossener Extraktion an den Füßen spielt bei allen Maßnahmen eine Hauptrolle. Der Zuzug eines Arztes ist dabei nicht verlangt, es sei denn, bei Verweigerung der Gebärenden und ihrer Umgebung den Eingriff von der Hebamme ausführen zu lassen. Nur dann soll sie eine Stunde nach abgeflossenem Fruchtwasser eine Führerin oder Kollegin rufen, gleichzeitig mit dem Hausarzt, einem «Geburtshelfer» oder «einem hierin erfahrenen Herrn Doktor». Auch hier wieder begegnen wir der bereits erwähnten ausdrücklichen Unterscheidung zwischen dem «Geburtshelfer» und «Herrn Doktor». Ist aber der Kopf schon tief ins Becken eingetreten, dann fällt natürlich die Wendung weg, trotzdem ist es nicht unbedingt notwendig, mit «scharfen Instrumenten 
gleich einzugreifen, sondern Ruhehaltung und Kraftarzneien haben schon oft gleichsam Wunder getan».

Über die Verwendung von Instrumenten spricht sich die HebammenOrdnung besonders eingehend aus. Zunächst handelt es sich offenbar lediglich um zerstückelnde Operationen zum «Zerschneiden, Zerbrechen, Einhacken und dergleichen, wobei das unschuldige Kind allemal um sein Leben und meistens auch um die heilige Taufe kommt». Deshalb wird allen Hebammen der Gebrauch solcher «scharfer» Instrumente allen Ernstes verboten, unter nochmaligem Appell an die nötige Geduld, die of tmals besser zum Ziele führe, wobei aber die frühzeitige Erkennung der Regelwidrigkeit und der Zuzug des Arztes wesentlich bleibt. Die Kontrolle der Herztöne kam damals natürlich noch nicht in Betracht, denn sie wurde erst siebzig Jahre später (1818) von Mayor (Genf) in die Praxis eingeführt. Allerdings muß er sich in die Priorität teilen mit Legoust, der schon 1650 die kindlichen Herztöne am Unterleib der Schwangern gehört haben soll, aber mit seiner Entdeckung nicht durchdrang. Zur Feststellung des eingetretenen Kindstodes wird deshalb die Hebamme auf das Pulsieren der Nabelschnur und der Fontanellen eventuell auch auf Mazarationserscheinungen beim vorliegenden Kindsteil verwiesen, wenn «ohne vorher gemachten Kraz oder allzuoftes Angreifen das Häutlein und am Kopf die Haare sich leichtlich abstreifen lassen».

Der Entbindung vermittels Instrumenten durch Accoucheurs ist ein besonderes eingehendes Kapitel gewidmet.

Als «sicheres und wohlnützbares» Instrument wird, ausschließlich zum Gebrauch der Accoucheurs, «die hier schon bekannte und mit Nutzen gebrauchte englische Zange und der zu Paris neu ersonnene und in Bologna verbesserte Kopfzieher» empfohlen. Der letztere findet eine genaue Beschreibung. Das Instrument sei von Paris nach Bologna dem Herrn Doktor Molinelli zugeschickt und vom Herrn Dr. Galli verbessert worden. Bei dem englischen Instrument hat man es offenbar mit dem von Peter Chamberlen dem Ältern angeblich um das Jahr 1600 angegebenen Modell zu tun. Hugh Chamberlen der Jüngere hat damit 1670 einen unglücklichen, drei Stunden dauernden Versuch gemacht und zwar am 19. August bei einer $38 \mathrm{j}$. Erstgebärenden mit Beckenenge. Der Exitus trat nach 24 Stunden ein. Jean Palfyn (Gent) 1723 gilt als Vater der später gebräuchlichen Zange. Bekannt ist weiter, daß Levret (Paris, 1705-1780) eine Verbesserung der bis dahin üblichen Modelle angegeben hat, mit Beckenkrümmung und sogenanntem französischem Schloß: Junctura per axin. 
Der in der Hebammen-Ordnung genannte Molinelli ist in der Geschichte der Geburtshilfe und speziell in der von Zweifel ausführlich gegebenen Geschichte der Zange nicht aufzufinden, wohl aber wird ein Doktor Gian Antonio Galli in Bologna ums Jahr 1757 genannt, der offenbar mit dem Vorerwähnten identisch sein dürfte. Er erhielt in jenem Jahr den Lehrstuhl für Geburtshilfe in Bologna und war berühmt durch seine Unterrichtsmodelle für Ärzte und Hebammen und durch ein geburtshilfliches Phantom.

Die Beschreibung des Instrumentes in der Augsburger HebammenOrdnung lautet folgendermaßen: «Das Pariser besteht aus drei, das verbesserte aber nur aus zwei stählernen Federn oder Schienen an einer bequemen Handhebe, deren ist jede bei neun Zoll lang und kaum über einen halben Zoll breit, biegsam, glatt, gar nicht schneidig, sondern mit Fleiß auf beiden Seiten glatt, stumpf und mugelicht geschliffen, nach der Breite oder Fläche auswärts gebogen und zwar in der Mitten ungefähr gegen zween Zoll breit, und so fort gegen oben und gegen unten zu verloren, ebenso viel wiederum einwärts gebogen, zu außerst an dem End rund und eine Schiene an die andere, zuvorderst vermittelst einer Niete und unterhalb bei der Handhebe vermittelst eines Wirbels zusammengefügt. Beide Schienen liegen so genau übereinander, als ob es nur eine einzige wäre, dergestalt, daß das Instrument dem von unten an langlecht ausgestreckten und oberher besser rundenhalben Bogen und der Handhebe nach aussiehet wie eine Rakete, die man zum Ballspielen gebraucht, wenn man diese von zuvorderst und zuoberst an teilte und so zerteilt, die eine Seite an der Handhebe stehen ließe, die andere aber bis zur Handhebe hinwegnähme. Die Zusammenfügung beider Schienen ist dermaßen eingerichtet, daß die eine Schiene vermittelst ihres Angels in der Handhebe fest und unbeweglich bleibt, die andere aber sich von der einen vermittels gemeltten Wirbels unten um den Angel und oben am End um den Nietstift herumbewegen läßt und so von einander hinweggeschoben, stellen alsdann beide Schienen miteinander gleichsam eine unzerteilt gelassene Ballspielrakete für. Einem Kind nun, das mit dem Kopf in der Geburt stecken geblieben, zur Welt zu helfen, bringt man dieses Instrument, beide Schienen übereinandergeschoben, zuerst hinter dem einen Ohr des Kindes bis unter sein Kinn, hernach schiebt man die andere Schiene, die sich von der einen hinwegschieben läßt über das selbige Ohr und über das Gesicht hin bis über das andere Ohr hinüber, alsdann ist das Köpflein in die Fläche beider Schienen wie in eine 
Schlinge eingefangen und kann also mit diesem Instrument unter dem Kinn an beiden untern Kinnbacken angefaßt, ohne mindeste Gefahr und Verletzung füglich und glücklich herausgezogen werden.»

Nach dieser Beschreibung unterscheidet sich das Molinellische Instrument, das nirgends in der Geschichte der Zange Erwähnung findet, hauptsächlich dadurch, daß die beiden schmalen Löffel einander mit ihren Konkavitäten anliegend eingeführt wurden und erst dann die innere Platte über den Schädel des Kindes wegwanderte, um so den Schädel von beiden Seiten zu fassen. Versager scheinen übrigens auch damit vorgekommen zu sein, denn auf sie wird ausdrücklich verwiesen und bei inzwischen mutmaßlicherweise abgestorbenem Kind die Applizierung der andern Instrumente vorgeschlagen, aber nur «mit Zuziehung und Rat noch eines andern in diesen Sachen erfahrenen Herrn Medici, auch mit vollkommener Einwilligung der Gebärerin und ihres Ehemannes und zumal mit aller menschenmöglichen Behutsamkeit und Gottesfürchtigkeit». Durch ein spezielles Ratsdekret vom 31. Juli 1749 wird übrigens von diesen Accoucheurs ein besonderes Examen vor Dekan und Vikar des Collegii Medici und den dazu deputierten Doktores in Theorie und Praxis verlangt.

Im Wochenbett hat die Hebamme neun Tage lang die Wöchnerin zu betreuen. Eine Temperaturmessung mittels Thermometer gab es natürlich noch nicht, um so strenger waren alle sonstigen Zeichen des Wohlbefindens oder fieberhafter Erkrankung zu beachten. Das Neugeborene soll sie gewissenhaft pflegen, Wiederbelebungsversuche energisch durchführen und gegebenenfalls die Nottaufe nach wörtlich aufgeführter Vorschrift erteilen, aber nur, wenn noch sichere Lebenszeichen vorhanden. Andernfalls wäre das ein "gottesverächtlicher und sträflicher Mißbrauch des heiligen Sakraments». Besonders ans Herz gelegt wird der Hebamme, daß nicht etwa einem Knäblein ein weiblicher oder einem Töchterlein ein Mannsname beigelegt, oder anstatt des natürlichen reinen Wassers etwa Wein, Bier oder vermischtes Wasser zum Taufen verwendet werde.

Im Kapitel Mißgeburten wird der gelegentlichen Schwierigkeit, das Geschlecht zu bestimmen, besondere Bedeutung beigemessen und im Zweifelsfalle Zuzug des Arztes und Anzeige an die Behörde zur Pflicht gemacht.

Das Auftreten bei der Taufe in der Kirche gestaltet sich für die Hebamme zum streng vorgeschriebenen Zeremoniell.

In Ehegerichtssachen soll die Hebamme der Obrigkeit, dem Reverendo 
Consistorio und dem Evangelischen Ehegericht begutachtend zur Seite stehen. Die ganze Kasuistik dieser Fragen wird im einzelnen erwähnt.

Strenge ist die Abtreibung geahndet, da «die Seele alsbald von der Empfängnis an vorhanden, sonst könnte das Kind nicht wachsen». Darum ist die Strafe dafür denn auch gemäß der peinlichen Halsgerichtsordnung Karls des V. sehr streng, ebenso für die vorsätzliche Sterilisierung von Mann oder Frau. Der schuldige Mann soll mit dem Schwert hingerichtet, die Frau ertränkt oder sonst «zum Tod» gestraft werden. Hat die Schwangerschaft noch nicht fünf Monate gedauert, so "pflegen die Urteiler der Strafe bei denen Rechtsverständigen Raths».

Die Sectio in Mortua wird zur strengsten Pflicht gemacht und zwar auch dann, wenn der Tod der Schwangern schon im vierten oder fünften Monat erfolgt, weil trotzdem mit einer eventuellen Rettung der Frucht zu rechnen sei.

Die Pflichten der vier Führerinnen werden besonders aufgezählt. Sie haben mit gutem Beispiel voranzugehen, üben die Aufsicht aus, kontrollieren die Lernenden, und die Älteste von ihnen stellt die Verbindung mit der Obrigkeit her. Führerinnen und Hebammen werden feierlich vereidigt, unter nochmaliger, neun Seiten langer Aufführung aller Pflichten. Die Lernerinnen legen zu Beginn des Kurses ein Handgelübde ab.

Endlich ist auch «die Bestallung der vier Ob-Frauen und zwei Herren Doktorum Medizinae, welche zur Hebammen-Ordnung verordnet sind,» genau geregelt. Sie bilden eine Art Aufsichtskommission und zwar muß immer die konfessionelle Vertretung zu gleichen Teilen streng gewahrt werden. Sie versammeln sich alle Vierteljahre zur Erledigung der bereits erwähnten Obliegenheiten. Die beiden Doktoren führen das Protokoll für je ihren Konfessionsteil. Alle eventuell eingelaufenen Klagen gegenüber Hebammen müssen strengstens geprüft und nötigenfalls Verweise und Strafen erteilt werden. Vergehen wider die Pflicht gegen Gott zieht Entlassung nach sich. Alle weitern Verstöße werden in 25 Artikeln mit dem jeweiligen Strafmaß aufgeführt.

Endlich folgt die Prüfungs- und Unterrichtsordnung für die Herren Doktores, die zu diesem Amt verordnet sind. Sie erteilen gegen Besoldung alle Monate ein paar Stunden Lektion und demonstrieren alle Jahre einmal an einem Leichnam eines «justifizierten Weibeskadavers» den Bau der Organe. Die Prüfung soll streng sein und darüber der Behörde Bericht erstattet werden. Als Entschädigung bezahlt die Schülerin jeder Ob-Frau und jedem Herrn Doktor zwei Gulden, zusammen sechs, der 
Lehrfrau zum Einstand und weiter für jedes Lernjahr einen Gulden, jedem der beiden Examinatoren je drei Gulden, für die Aufnahme in die eigentliche Hebammenklasse dem Herrn Doktor zwei Gulden und den Ob-Frauen und Führerinnen ihrer Religion zusammen vier Gulden, für die Vorstellung zur Besoldung dem Herrn Doktor zwei Gulden und bei der Ernennung zur Führerin dem Doktor zwei Gulden und jeder ObFrau einen Gulden. Es bestand also bereits damals eine Art Schulgeld und, bei Umwertung der Werte, im Vergleich zu heute ein gar nicht so bescheidenes.

Der diesem Aufsatz gezogene Rahmen erlaubte leider manches interessante Detail nur andeutungsweise zu streifen. Aber auch aus dieser gedrängten Zusammenstellung ergibt sich die große Bedeutung dieser Berufsregelung, die schon vor zweihundert Jahren eine weitsichtige Behörde veranlaßte, Ausbildung und Berufstätigkeit der Hebamme genau zu regeln und zu fördern. Wenn wir bedenken, wie geringschätzig vielfach der Hebammenberuf in der Folgezeit von den Behörden, zum Teil bis in unsere jüngste Gegenwart, behandelt wurde, so kann man von der Augsburger Hebammen-Ordnung wohl behaupten, daß sie ein einzig dastehendes Dokument darstellt.

P. Jung, St. Gallen

\section{Buchbesprechungen}

Nova Acta Paracelsica, II. Jahrbuch d. schweiz. Paracelsus-Gesellschaft, 1945, Verlag Birkhäuser, Basel, $199 \mathrm{~S}$.

Auch dieser zweite, von der schweiz. Paracelsus-Gesellschaft herausgegebene Sammelband enthält wieder eine stattliche Reihe von Paracelsusstudien, naturgemäß von unterschiedlichem Wert. K. Bittel behandelt «Die Basler Lektur des Theophrast", ein Thema, das schon A. Burckhardt und Karcher behandelt haben. In gewohnt sorgfältiger Weise wird festgehalten, was historisch verbürgt ist; da die Überlieferung lückenhaft ist, sind Konjekturen noch immer unvermeidlich. Bittel nimmt an, Paracelsus habe in Basel auf einem freien Lehrstuhl, «außerhalb der Fakultät» gelesen, «außerhalb der Konstitution der Universität». An sich ist diese Interpretation durchaus möglich und würde manche Schwierigkeit zwanglos erklären. Solche freie Lekturen gab es auch für die Theologen, da die vom neugläubigen Rat 1523 abgesetzten Professoren die altgläubige Hochschule erst 1529 verließen (A. Burckhardt, Gesch. d. med. Fakult. zu Basel, S. 18). - Basilio de Telepnef steuert einen Beitrag über "Paracelsus auf den deutschen Hochschulen» bei. Die mangelhafte historische 